\title{
Transient impact of a liquid column on a miscible liquid surface
}

\author{
B. Kersten, C. D. Ohl, and A. Prosperetti ${ }^{\mathrm{a})}$ \\ Department of Applied Physics, Twente Institute of Mechanics and Burgerscentrum, Department of Applied \\ Physics, University of Twente, AE 7500 Enschede, The Netherlands
}

(Received 10 October 2002; accepted 3 December 2002; published 5 February 2003)

\begin{abstract}
The flow induced by a liquid column falling on an undisturbed liquid surface is studied with the aid of a high-speed camera. The falling liquid spreads over the receiving liquid forming a cavity which eventually pinches off due to the action of gravity. It is only at this point that the normal flow pattern in which the impacting liquid penetrates below the free surface is established. The same process-at a scale smaller by four orders of magnitude-is encountered in the jetting behavior of collapsing cavitation bubbles. It is also observed that the cavity dynamics is strikingly similar to that found when a disturbance is induced on a steady jet falling on a liquid. This observation supports a generic mechanism for air entrainment hypothesized in an earlier paper. (C) 2003 American Institute of Physics. [DOI: 10.1063/1.1542614]
\end{abstract}

At sufficiently large Reynolds number, the streamlines of a jet impinging steadily on the surface of a pool of a miscible liquid separate from the free surface and (aside from the effect of well-known mixing and instability mechanisms) penetrate into the pool in the same general direction as the jet. This flow pattern is quite different from that predicted by the inviscid theory, according to which the jet fluid would spread over the pool fluid without penetrating it. The latter behavior is in fact observed in the case of a drop: it has been known at least since the classic work of Worthington, ${ }^{1}$ that the drop liquid initially spreads along the surface, and only later penetrates below it and mixes with the receiving liquid.

In this Brief Communication we show that, for a sufficiently high-speed jet, the reconciliation of the irrotational flow prediction and the observed steady flow is intimately related to the mechanism by which the jet entrains air when it first strikes the free surface. Furthermore, the present results confirm a generic mechanism for air entrainment in free-surface flow recently postulated in connection with a somewhat different experiment. ${ }^{2}$

We carried out a simple experiment in which a 4-mmdiam column of water falls vertically on a quiescent water surface in a small tank (height $\times$ width $\times$ breadth $=0.19$ $\left.\times 0.19 \times 0.29 \mathrm{~m}^{3}\right)$. A glass tube $(0.2 \mathrm{~m}$ in length with a $4 \mathrm{~mm}$ inner diameter) is filled with water and the upper opening of the tube closed with the thumb. The diameter is small enough that surface tension stabilizes the liquid surface at the tube exit and prevents the water from falling. The tube is positioned vertically above the pool surface, the thumb lifted, and the liquid column falls onto the pool. The ensuing process is observed with a high-speed camera (Imager CR 1000, Roper Scientific) at a frame rate of $1000 \mathrm{fps}$ under diffusive backillumination. By comparing sequences taken under nominally identical conditions it was found that, in spite of the simplicity of the procedure, the flow generated

a)Permanent address: Department of Mechanical Engineering, The Johns Hopkins University, Baltimore, MD 21218. was quite reproducible. In some of the experiments, the water was colored with blue ink.

Figure 1 shows a typical sequence of events. The liquid column enters the pool and generates a cavity which then collapses from the sides entrapping a toroidal bubble. Here the Froude number, defined by $\mathrm{Fr}=U^{2} / g d$ ( $U$ impact velocity of the water column, $d$ tube diameter, $g$ acceleration of gravity), is 36.7 ; the Weber $\mathrm{We}=\rho d U^{2} / \sigma$ ( $\rho$ liquid density, $\sigma$ surface tension coefficient) and Reynolds number Re $=\rho d U / \mu$ ( $\mu$ viscosity coefficient $)$ are 79 and 4800 , respectively. A sequence taken in very similar conditions (Fr $=39.2, \mathrm{We}=84, \mathrm{Re}=4960$ ), but with the addition of ink to the water, is shown in Fig. 2. Here the cavity appears thicker. It is revealing to look at the images obtained by subtracting pixel by pixel the digitized gray levels of the two sets of images (Fig. 3). These processed images clearly show that the dyed fluid remains attached to the surface of the cavity, as predicted by irrotational flow theory.

The results of a similar subtraction in which the dyed fluid images were obtained with $\mathrm{Fr}=34.9, \mathrm{We}=75, \mathrm{Re}$ $=4680$ are shown in Fig. 4. It is seen here that, after the toroidal bubble at the bottom of the cavity pinches off and the whole cavity collapses against the jet, the separated flow pattern described at the beginning and associated with a steady jet flow is established. We have observed the same process every time the impact velocity of the liquid column was large enough to generate a cavity and entrain air. In our experiments the threshold for this occurrence corresponded to a Froude number of about 10. At lower Froude numbers the entrapped bubble was very small or nonexistent and the stagnation pressure insufficient to deflect the incoming liquid stream. A sequence with $\mathrm{Fr}=3.2, \mathrm{We}=6.8$, and $\mathrm{Re}=1412$ is shown in Fig. 5.

Reference 3 presented the results of a similar experiment, which differed in that the mass of falling water was much greater although the Froude number was in a comparable range. In that study it was argued that, for sufficiently high Froude numbers $\mathrm{Fr}_{*}$ (based on the free-fall velocity $U_{*}$ 

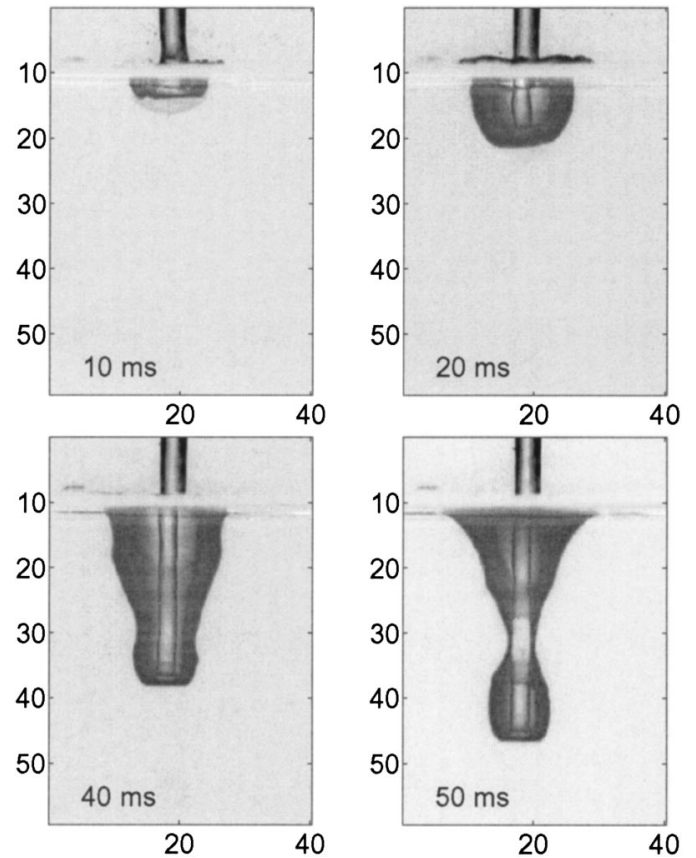

FIG. 1. Four frames from a $1000 \mathrm{fps}$ movie showing the impact of a liquid column onto a quiescent miscible liquid surface. The Froude, Weber, and Reynolds numbers are 36.7, 79, and 4800, respectively.

of the liquid column center-of-mass), the closure time $t_{c}$ of the cavity is approximately given by $6(d / 2 g)^{1 / 2} \mathrm{Fr}_{*}^{-1 / 6}$, and the cavity depth below the undisturbed free surface at the moment of closure was $H_{c}=\frac{3}{2} d \mathrm{Fr}_{*}^{1 / 3}$. For the cases of Figs. 1 and 2 , the measured values are $t_{c}=49 \mathrm{~ms}, 50 \mathrm{~ms}$, and $H_{c}=35.9 \mathrm{~mm}, 35.1 \mathrm{~mm}$. If the Froude number is based on the measured impact velocity, rather than $U_{*}$, these formulas give $47 \mathrm{~ms}, 47 \mathrm{~ms}, 47 \mathrm{~ms}$, and $19.6 \mathrm{~mm}, 19.9 \mathrm{~mm}$. We found
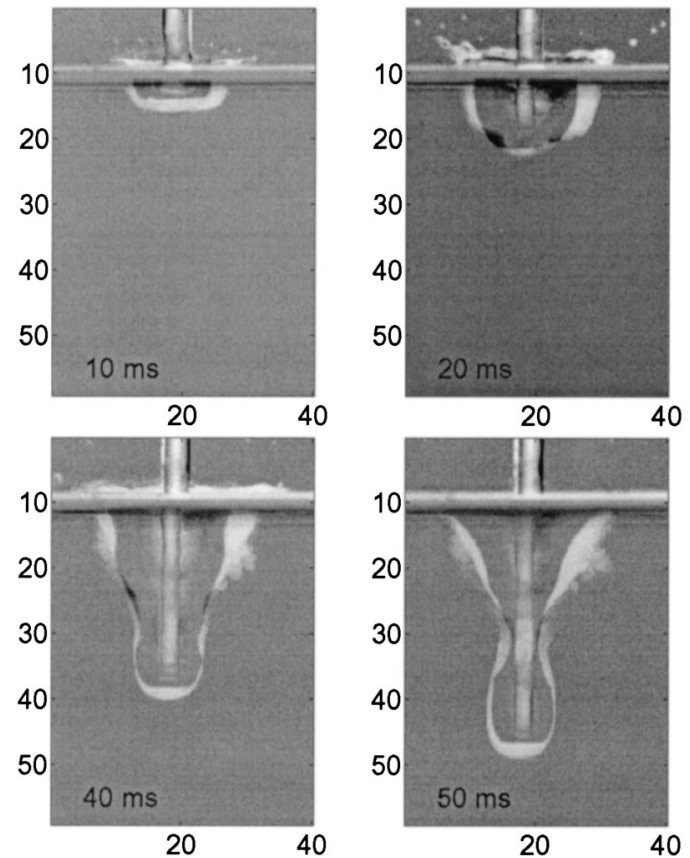

FIG. 3. The result of a pixel-by-pixel digital subtraction of the images in Figs. 1 and 2. Note the clear localization of the colored liquid along the surface of the cavity, evidence of the attached nature of the streamlines.

that in our case the measured impact velocity was lower than the free-fall velocity, probably due to viscous effects in the tube. In any event, a reconciliation of the present data with the previous cavity depth correlation would require an adjustment of the velocity of a factor of about 2.4, which is unrealistic and would, furthermore, lead to a bad estimate for $t_{c}$. Furthermore, inviscid theory predicts that, in the absence of gravity and unsteadiness, the velocity $V_{c}$ at which the
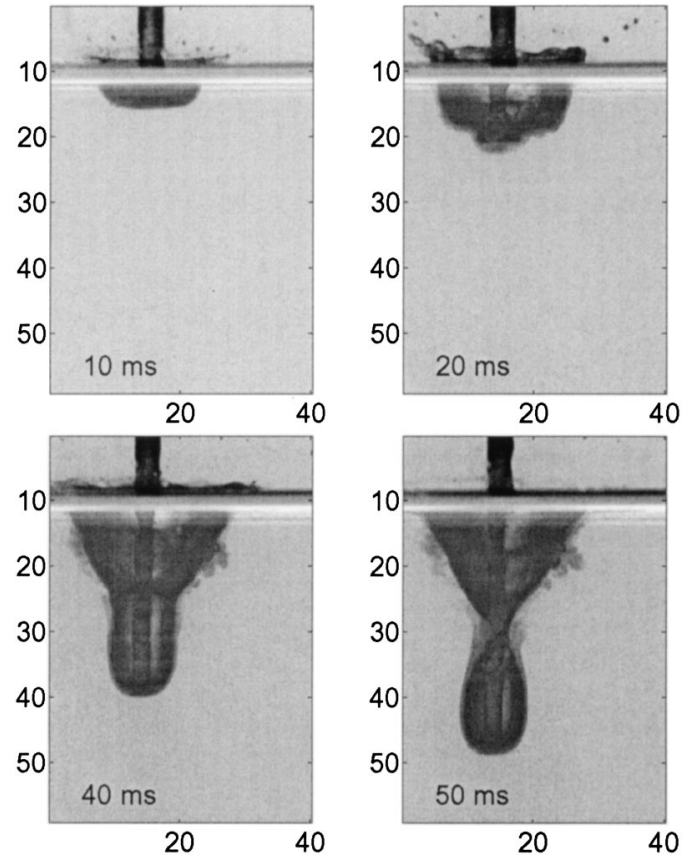

FIG. 2. As in Fig. 1 with $\mathrm{Fr}=39.2$, $\mathrm{We}=84$, and $\mathrm{Re}=4960$, respectively. The jet liquid is dyed with ink.
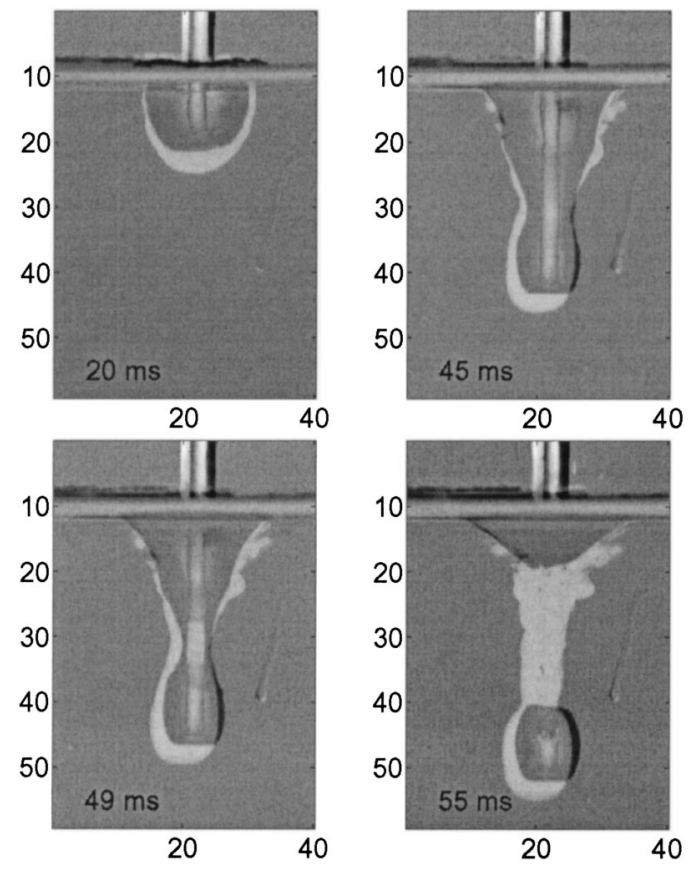

FIG. 4. As in Fig. 3. The dyed images subtracted correspond to an experiment with $\mathrm{Fr}=34.9, \mathrm{We}=75$, and $\mathrm{Re}=4680$, respectively. The last frame shows that the jet streamlines have separated from the free surface. 

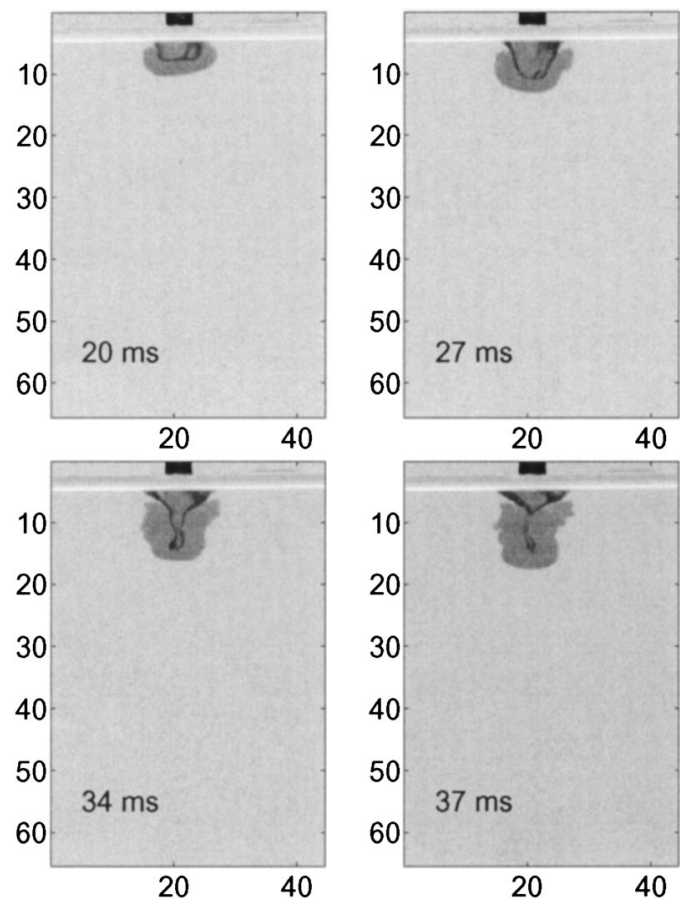

FIG. 5. As in Fig. 2 with $\mathrm{Fr}=3.2, \mathrm{We}=6.8$, and $\mathrm{Re}=1412$. In this case the stagnation pressure is not sufficient to deflect the falling liquid.

bottom of the cavity penetrates the liquid pool is half of the jet velocity $U .{ }^{4,5}$ For the cases of Figs. 1 and 2 we have $U$ $=1.20 \mathrm{~m} / \mathrm{s}, 1.17 \mathrm{~m} / \mathrm{s}, V_{c}=0.82 \mathrm{~m} / \mathrm{s}, 0.72 \mathrm{~m} / \mathrm{s}$, respectively, with $V_{c} / U=0.68$ and 0.62 .

In the present experiment, surface tension caused the formation of a blob at the front of the column which was not present in the experiments of Ref. 3. To investigate whether this feature was at the root of the observed differences, we carried out another series of tests in which the liquid fell inside the tube except for the last $10 \mathrm{~mm}$. With this arrangement, the surface-tension induced artifact was much reduced, but the cavity depth was still underestimated by a factor of approximately 2 and $V_{c} / U$ was between 0.7 and 0.9 . The smaller diameter of the impacting jet front also resulted in slightly thinner and deeper cavities.

The major difference between the present study and that of Ref. 3 was the diameter of the jet, which here was 1 to 2 orders of magnitude smaller resulting, in particular, in comparable differences in the Weber and Reynolds numbers. The observations therefore suggest a strong effect of these parameters, in spite of the fact that, in the present study, their value was already relatively large (about 80 and 5000, respectively). We estimate that, before leaving the tube, the liquid had nearly reach fully developed flow conditions. This circumstance would suggest, in particular, that the velocity distribution in the impacting slug has an effect.

It is interesting to point out the occurrence of the same process in a phenomenon that occurs at spatial scales smaller by many orders of magnitude (Fig. 6). It is well known that very often collapsing cavitation bubbles develop jets which strike the opposing bubble wall with velocities that can be as high as hundreds of meters per second. ${ }^{6-8}$ These liquid micro-jets, with typical diameters of the order of microme-
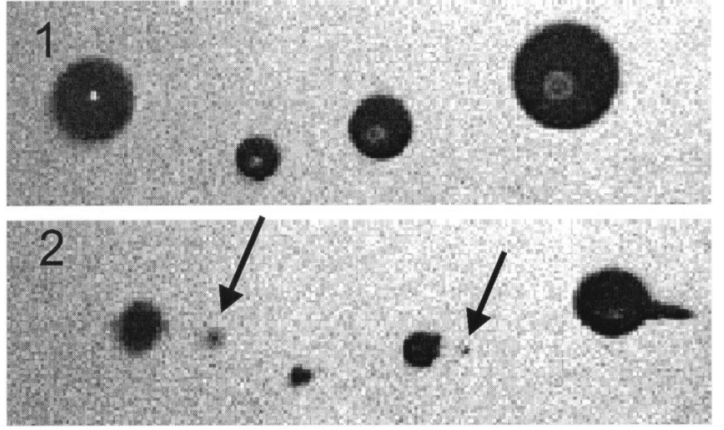

FIG. 6. Four electrolytically generated oxygen bubbles (the largest one with a diameter of $96 \mu \mathrm{m}$, the smallest one of $40 \mu \mathrm{m}$ ) before (upper frame) and just after being subjected to a $\sim 20 \mathrm{MPa}$ shock wave propagating from left to right. In the lower frame, the largest bubble exhibits a gas-surrounded jet developed during the shock-induced-collapse and directed along the direction of propagation of the shock. The smaller bubbles have been hit by the shock earlier and have already collapsed. The tiny bubbles marked by the arrows are the remnants of their jets which have broken off due to surface tension effects. The process is analogous to that observed in the last frame of Figs. 1 and 2. Illumination was provided by a $150 \mathrm{~ns}$ flash which stops the motion. Each frame has a size of $182 \times 528 \mu \mathrm{m}^{2}$.

ters or less, do not pierce the bubble wall, but form a cavity similar to that shown in Figs. 1 and 2-it is actually the gas "shroud" surrounding the jet which makes the process visible. Later, due to the effect of surface tension, this structure pinches off to form a tiny, presumably toroidal, bubble with a qualitative similarity to the last frame of our Fig. 1. Vogel et al. ${ }^{9}$ have shown that the liquid in the jet does not pierce the bubble wall, but remains confined along the free surface of the developing structure.

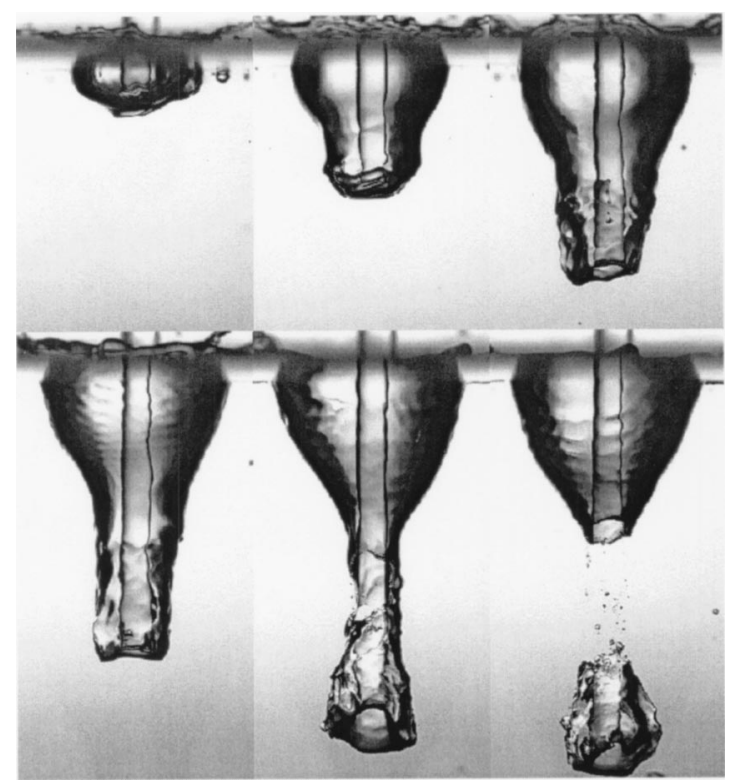

FIG. 7. The sequence of events induced by a surface disturbance (shown in Fig. 8) on a steady jet (from Ref. 2). The process of cavity formation and air entrainment bear a strong similarity to the sequences shown in Figs. 1-4, in spite of the fact that, unlike those cases, the jet is here continuous, rather than freshly impacting the free surface. The similarity between the two phenomena implies that the streamlines of the steady jet reattach to the surface of the pool when the disturbance they carry strikes the surface, as observed in Figs. 3 and 4. 


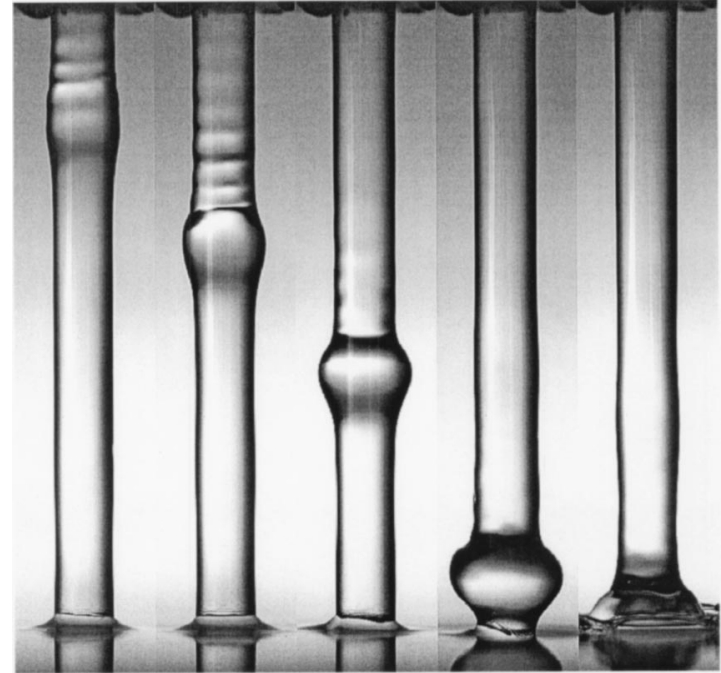

FIG. 8. The jet disturbance responsible for the process shown in Fig. 7 (from Ref. 2).

Another interesting result of this study can be found by comparing Figs. 1 and 2 with Fig. 7, taken from Ref. 2. The similarity of the cavity shapes is striking, and becomes quite unexpected when it is realized that the two experimental situations are actually very different. In the experiment of Ref. 2 there is a steady, nonentraining jet with streamlines separating at the free surface. The cavity is formed by inducing on this jet a disturbance, shown in Fig. 8 (also from the same reference), which strikes the water surface with the effect shown in Fig. 7. The key to the similarity between the two situations is very likely to be found in a hypothesis set forth in Ref. 2, namely that the stagnation pressure generated when the disturbance carried by the steady jet strikes the pool surface causes a momentary reattachment of the streamlines to this surface. Thus, the disturbed jet behaves as if it were freshly impacting on the surface-as in the present experiment-unaffected by the preexisting steady flow situation. That this process is possible is suggested by the fact that the pressure satisfies a Poisson equation, which implies that the characteristic length scale for pressure variations is set by the geometry of the boundary and is therefore, for the experiments of Ref. 2, of the order of the jet radius. The implication is that, when the surface disturbance strikes free surface, the pressure is fairly uniform-and therefore close to the stagnation pressure-over the jet cross section. While the present results do not constitute an irrefutable proof of this hypothesis, they are at least strongly supportive of it.
If this mechanism is accepted, its implications for air entrainment in nominally steady flows (e.g., a waterfall, or a steady jet) are significant. In such flows, the surface of the falling liquid is usually strongly disturbed by turbulence. When the crests of these disturbances strike the pool liquid, they generate cavities which close and entrap air. As observed in Ref. 2, if the only energy available were the kinetic energy of the surface disturbance, the cavity would not become very deep and no air could be entrained. The reattachment of the streamlines, however, has the effect that part of the jet energy is made available to increase the cavity depth so that air entrainment becomes possible. This process of flow reattachment appears therefore to be a generic mechanism contributing - with others, see, e.g., Refs. 10-13-to air entrainment in free-surface flows.

\section{ACKNOWLEDGMENT}

This study has been supported by the Fundamenteel Onderzoek der Materie of The Netherlands under Grant No. 99MFS06.

${ }^{1}$ A. M. Worthington, A Study of Splashes (Longman \& Green, London, 1908; reprinted by Macmillan, New York, 1963).

${ }^{2}$ Y. G. Zhu, H. N. Oguz, and A. Prosperetti, "Air entrainment by impinging liquid jets with surface disturbances," J. Fluid Mech. 404, 151 (2000); see also Phys. Fluids 10, S3 (1998).

${ }^{3}$ H. N. Oguz, A. Prosperetti, and A. R. Kolaini, "Air entrapment by a falling water mass," J. Fluid Mech. 294, 181 (1995).

${ }^{4}$ G. Birkhoff and E. H. Zarantonello, Jets, Wakes, and Cavities (Academic, New York, 1957).

${ }^{5}$ H. N. O guz, A. Prosperetti, and A. M. Lezzi, "Examples of air-entraining flows," Phys. Fluids A 4, 649 (1992).

${ }^{6}$ W. Lauterborn and H. Bolle, "Experimental investigations of cavitationbubble collapse in the neighborhood of a solid boundary," J. Fluid Mech. 72, 391 (1975).

${ }^{7}$ N. K. Bourne and J. E. Field, "Shock-induced collapse of single cavities in liquids," J. Fluid Mech. 244, 225 (1992).

${ }^{8}$ A. Philipp and W. Lauterborn, "Cavitation erosion by single laserproduced bubbles," J. Fluid Mech. 361, 75 (1998).

${ }^{9}$ A. Vogel and W. Lauterborn, "Untersuchung nichtsphärisch kollabierender Kavitations-blasen mit einem Raumfrequenzfilterungsverfahren," in Fortschritte der Akustik-DAGA '84 (Proceedings of the 1984 German Acoustical Society Meeting), Darmstadt, 26-29 March 1984 (Bad Honnef, DPG-GmbH, 1984), pp. 481-484.

${ }^{10}$ E. Van de Sande and J. M. Smith, "Surface entrainment by high velocity water jets," Chem. Eng. Sci. 28, 1161 (1973).

${ }^{11}$ E. Van de Sande and J. M. Smith, "Jet break-up and air entrainment by low velocity turbulent water jets," Chem. Eng. Sci. 31, 219 (1976).

${ }^{12}$ A. K. Biń, "Gas entrainment by plunging liquid jets," Chem. Eng. Sci. 48, 3585 (1993).

${ }^{13} \mathrm{H}$. Chanson, Air Bubble Entrainment in Free-surface Turbulent Shear Flows (Academic, San Diego, 1996). 